CERN-TH/98-320

DEMO-HEP/98-04

hep-th/9810042

\title{
OCTONIONIC GRAVITATIONAL INSTANTONS
}

\author{
I. Bakas ${ }^{a}$, E.G. Floratos ${ }^{b, c}$ and A. Kehagias ${ }^{d^{*}}$ \\ ${ }^{a}$ Department of Physics, University of Patras, GR-26500, Greece \\ ${ }^{b}$ Institute of Nuclear Physics, NRCS Democritos, Athens, Greece \\ ${ }^{c}$ Physics Department, University of Crete, Iraklion, Greece \\ ${ }^{d}$ Theory Division, CERN, 1211 Geneva 23, Switzerland
}

\begin{abstract}
We construct eight-dimensional gravitational instantons by solving appropriate selfduality equations for the spin-connection. The particular gravitational instanton we present has $\operatorname{Spin}(7)$ holonomy and, in a sense, it is the eight-dimensional analog of the Eguchi-Hanson 4D space. It has a removable bolt singularity which is topologically $S^{4}$ and its boundary at infinity is the squashed $S^{7}$. We also lift our solutions to ten and eleven dimensions and construct fundamental string and membrane configurations that preserve $1 / 16$ of the original supersymmetries.
\end{abstract}

*e-mails: bakas@nxth04.cern.ch, bakas@ajax.physics.upatras.gr

manolis@timaios.nrcps.ariadne-t.gr

alexandros.kehagias@cern.ch

CERN-TH/98-320

October 1998 


\section{Introduction}

The main purpose of the present work is to construct an eight-dimensional analogue of the non-compact gravitational instantons that arise in four dimensions [1, 22, 3], the simplest example being the Eguchi-Hanson space [4]. It is well known that in four dimensions the non-trivial structure of such gravitational instantons is encoded into the asymptotic form

of the metric whose boundary structure at infinity is given by the lens spaces of $S^{3}$. Since $S^{3}$ is the Hopf fibration of $S^{1}$ over $S^{2}$ it is quite natural to employ the topological structure of the seven-sphere, $S^{7}$, as Hopf fibration of $S^{3}=S U(2)$ over $S^{4}$ in our search of eightdimensional gravitational instantons. In the context of gauge fields the first Hopf fibration arises in the construction of the $U(1)$ Dirac monopole with unit magnetic charge, while the second Hopf fibration arises in the construction of the $S U(2)$ Yang-Mills instanton with unit topological number [5]. Because of this analogy we expect that self-dual $S U(2)$ YangMills configurations in four dimensions will play an interesting role in future investigations of eight-dimensional gravitational instantons, for instance in generalizing the notion of nut potential in eight dimensions and classifying the fixed point sets of their isometry groups. We will make a few remarks in this direction at the very end of the paper.

In any event the solution we present here could be viewed as the simplest non-trivial example of a more general class of eight-dimensional gravitational instantons, but further results will be reported elsewhere. The metric we construct in the sequel has the squashed seven-sphere (as opposed to the round seven-sphere) as its boundary space at infinity, which is essential for establishing the non-triviality of our solution in eight dimensions. Alternatively we could have arrived at the same result by deforming the squashed sevensphere in the interior of an eight dimensional space using appropriately chosen coefficient functions, but we decided to emphasize the aspects of Hopf fibration in our presentation in view of other future generalizations.

As we have shown in [6], eight-dimensional manifolds which satisfy an appropriate self- 
duality condition for the spin connection have holonomy in $\operatorname{Spin}(7)$. This provides us with a systematic way of constructing $\operatorname{Spin}(7)$ holonomy manifolds by solving appropriate firstorder equations. Non-compact such manifolds have first been constructed in [7] and further discussed in [8], while compact ones were constructed as $T^{8}$ orbifolds in [9] after appropriate resolution of the singularities. Here, by solving the eight-dimensional self-duality condition we find a space with $\operatorname{Spin}(7)$ holonomy and isometry group $S O(5) \times S U(2)$. It has a bolt singularity which is topologically $S^{4}$ and its boundary at infinity is the squashed $S^{7}$. Finally, we lift our solution in ten and eleven dimensions and we describe fundamental string and membrane configurations, which we name octonionic, and which preserve 1/16 of the original supersymmetries.

\section{Octonionic Algebra}

We will recall here some properties of the octonionic (Cayley) algebra $\mathcal{O}$ and its relation to $S O(8)$ following mainly [10, 11]. The octonionic algebra is a division algebra, which means it has a non-degenerate quadratic form $Q$ that satisfies $Q(x y)=Q(x) Q(y)$ and in addition $Q(x)=0$ implies $x=0$. The other division algebras are the real $\mathcal{R}$, complex $\mathcal{C}$ and quaternionic $\mathcal{H}$ algebras. $\mathcal{R}, \mathcal{C}$ are commutative, $\mathcal{H}$ is non-commutative but associative, while $\mathcal{O}$ is neither commutative nor associative. A basis for $\mathcal{O}$ is provided by the eight elements

$$
1, e_{a}, \quad a=1, \ldots, 7
$$

which satisfy the relation

$$
e_{a} e_{b}=\psi_{a b c} e_{c}-\delta_{a b}
$$

The tensor $\psi_{a b c}$ is totally antisymmetric with

$$
\psi_{a b c}=+1 \quad \text { for } \quad a b c=123,516,624,435,471,673,572
$$


We may also define its dual $\psi_{a b c d}$ as

$$
\psi_{a b c d}=\frac{1}{3 !} \epsilon_{a b c d f g h} \psi^{f g h}
$$

so that

$$
\psi_{a b c d}=+1 \quad \text { for } \quad a b c d=1245,2671,3526,4273,5764,6431,7531 .
$$

They satisfy the relations

$$
\begin{aligned}
\psi^{a b c} \psi_{d h c} & =\delta_{d}^{a} \delta_{h}^{b}-\delta_{h}^{a} \delta_{d}^{b}-\psi_{d h}^{a b} \\
\psi^{a b c d} \psi_{e h c d} & =4\left(\delta_{e}^{a} \delta_{h}^{b}-\delta_{h}^{a} \delta_{e}^{b}\right)-2 \psi_{e h}^{a b} \\
\psi^{a b c} \psi_{d e b c} & =-4 \psi_{d e}^{a} .
\end{aligned}
$$

Let us note here that the relation (2) is similar to the one obeyed by the quaternions (Pauli matrices). However, the latter satisfy the Jacobi identity as a result of the associativity of the quaternionic algebra, while the octonions $e_{a}$ are not associative and hence do not satisfy the Jacobi identity.

The tensor $\psi_{a b c}$ can be assigned to an $S O(8)$ representation $\Psi_{\alpha \beta \gamma \delta}$, where the Greek indices range from 1 to 8 and also set the notation $\alpha=(a, 8)$. We have

$$
\Psi_{\alpha \beta \gamma 8}=\psi_{a b c}, \quad \Psi_{\alpha \beta \gamma \delta}=\psi_{a b c d}
$$

which is self-dual

$$
\Psi_{\alpha \beta \gamma \delta}=\frac{1}{4 !} \epsilon_{\alpha \beta \gamma \delta \zeta \eta \theta \kappa} \Psi^{\zeta \eta \theta \kappa}
$$

and belongs to one of the three different 35 's of $S O(8) \mathbf{3 5 _ { v } ,} \mathbf{3 5}_{ \pm}$(related by triality). It satisfies the fundamental identity

$$
\begin{aligned}
\Psi_{\alpha \beta \gamma \delta} \Psi^{\zeta \eta \theta \delta}= & \left(\delta_{\alpha}^{\zeta} \delta_{\beta}^{\eta}-\delta_{\beta}^{\zeta} \delta_{\alpha}^{\eta}\right) \delta_{\gamma}^{\theta}+\left(\delta_{\alpha}^{\theta} \delta_{\beta}^{\zeta}-\delta_{\beta}^{\zeta} \delta_{\alpha}^{\theta}\right) \delta_{\gamma}^{\eta}+\left(\delta_{\alpha}^{\eta} \delta_{\beta}^{\theta}-\delta_{\beta}^{\theta} \delta_{\alpha}^{\eta}\right) \delta_{\gamma}^{\eta}+ \\
& \Psi_{\alpha \beta}{ }^{\zeta \eta} \delta_{\gamma}^{\theta}+\Psi_{\alpha \beta}{ }^{\theta \zeta} \delta_{\gamma}^{\eta}+\Psi_{\alpha \beta}{ }^{\eta \theta} \delta_{\gamma}^{\zeta}+\Psi_{\gamma \alpha}{ }^{\zeta \eta} \delta_{\beta}^{\theta}+\Psi_{\gamma \alpha}{ }^{\theta \zeta} \delta_{\beta}^{\eta}+ \\
& \Psi_{\gamma \alpha}{ }^{\eta \theta} \delta_{\beta}^{\zeta}+\Psi_{\beta \gamma}{ }^{\zeta \eta} \delta_{\alpha}^{\theta}+\Psi_{\beta \gamma}{ }^{\theta \zeta} \delta_{\alpha}^{\eta}+\Psi_{\beta \gamma}{ }^{\eta \theta} \delta_{\alpha}^{\zeta} .
\end{aligned}
$$


We may use the octonions $e_{a}$ to construct a representation of the $S O(7) \gamma$-matrices according to

$$
\left(\gamma_{a}\right)_{b c}=i \psi_{a b c}, \quad\left(\gamma_{a}\right)_{b 8}=i \delta_{a b}, \quad\left\{\gamma_{a}, \gamma_{b}\right\}=2 \delta_{a b}
$$

so that $\gamma^{a b}=\frac{1}{2}\left[\gamma^{a}, \gamma^{b}\right]$ are the $S O(7)$ generators. We may also form the $S O(8) \gamma$-matrices $\Gamma_{\alpha}=\left(\Gamma_{a}, \Gamma_{8}\right), \alpha=1, \ldots, 8$

$$
\Gamma_{a}=\left(\begin{array}{cc}
0 & i \gamma_{a} \\
-i \gamma_{a} & 0
\end{array}\right), \quad \Gamma_{8}=\left(\begin{array}{cc}
0 & 1 \\
1 & 0
\end{array}\right), \quad\left\{\Gamma_{\alpha}, \Gamma_{\beta}\right\}=2 \delta_{\alpha \beta},
$$

that correspond to the standard embedding of $S O(7)_{v}$ in $S O(8)$. The latter is defined as the stability subgroup $S O(7) \subset S O(8)$ of the vector representation according to which we have the decomposition

$$
8_{v}=7+1, \quad 8_{ \pm}=8
$$

where $\boldsymbol{8}_{v}, \mathbf{8}_{ \pm}$are the vector and the two spinorial representations of $S O(8)$. The $S O(8)$ generators $\Gamma^{a b}=\frac{1}{2}\left[\Gamma^{a}, \Gamma^{b}\right]$ satisfy the relations

$$
\Gamma^{a b}=\psi^{a b c} \Gamma^{9} \Gamma_{8 c}, \quad \psi_{a b c d} \Gamma^{a b}=-\left(4+2 \Gamma^{9}\right) \Gamma_{c d},
$$

where, as usual, $\Gamma^{9}=\left(\begin{array}{cc}1 & 0 \\ 0 & -1\end{array}\right)$ is the chirality matrix. It is not then difficult to verify that the generators

$$
G^{\alpha \beta}=\frac{3}{8}\left(\Gamma^{\alpha \beta}+\frac{1}{6} \Psi_{\gamma \delta}^{\alpha \beta} \Gamma^{\gamma \delta}\right)
$$

leave the right-handed spinor $\eta_{+}$invariant

$$
G^{\alpha \beta} \eta_{+}=0
$$

The stability group of $\eta$ is again another $S O(7)$, which we will denote by $S O(7)^{+}$, according to which

$$
8_{v}=8, \quad 8_{+}=7+1,8_{-}=8
$$


The singlet in the decomposition of $\mathbf{8}_{+}$corresponds to the null eigenspinor $\eta$ of $G^{\alpha \beta}$ in eq.(15). This can be seen by considering the Casimir $G^{\alpha \beta} G_{\alpha \beta}$ which has a zero eigenvalue for the $\mathbf{8}_{+}$. If we had used in eq.(14) the second $\mathbf{3 5}$ of $S O(8)$, the antiself-dual one, then $G^{\alpha \beta}$ would annihilate the left-handed spinor $\eta_{-}$. In this case, the stability group of the latter is a third $S O(7)^{-}$subgroup of $S O(8)$ defined by

$$
8_{v}=8,8_{+}=8,8_{-}=7+1
$$

Again, $G^{\alpha \beta}$ constructed with the antiself-dual 35 has a zero eigenvalue which corresponds to the singlet in $\mathbf{8}_{-}$. The three different $S O(7)$ subgroups of $S O(8), S O(7)_{v}, S O(7)^{ \pm}$are related by triality.

\section{3. $\operatorname{Spin}(7)$-Holonomy Spaces}

Let us now assume that the eight-dimensional spin manifold $X$ admits a spin connection which satisfies the self-duality condition

$$
\omega_{\alpha \beta \mu}=\frac{1}{2} \Psi_{\alpha \beta \gamma \delta} \omega_{\mu}^{\gamma \delta}
$$

where $\Psi_{\alpha \beta \gamma \delta}$ is defined in eq.(7). It is not difficult to verify that a relation of the form $\omega_{\alpha \beta \mu}=\lambda \Psi_{\alpha \beta \gamma \delta} \omega_{\mu \delta}^{\gamma \delta}$ is consistent for $\lambda=1 / 2,-1 / 6$ as can be verified by multiplying both sides by $\Psi^{\alpha \beta \zeta \eta}$ and using eq.(9). Here we consider only the case $\lambda=1 / 2$, while the other possibility has not been investigated and left to future work. Eq.(18) is an eight-dimensional analog of the standard self-duality condition in four dimensions where the totally antisymmetric symbol $\epsilon_{a b c d}$ is replaced by $\Psi_{\alpha \beta \gamma \delta}$. Eight-dimensional manifolds with a connection satisfying eq.(18) in the Euclidean regime will be called gravitational instantons. Note that the octonions have also appeared in the construction of an eightdimensional Kerr metric [12. The holonomy group of such manifolds is in $\operatorname{Spin}(7)$ as can be seen as follows. Recall first that a manifold is of $\operatorname{Spin}(7)$ holonomy if and only if the 
Cayley four-form [13]

$$
\begin{aligned}
\Psi= & e^{1} \wedge e^{2} \wedge e^{3} \wedge e^{8}+e^{5} \wedge e^{1} \wedge e^{6} \wedge e^{8}+e^{6} \wedge e^{2} \wedge e^{4} \wedge e^{8}+e^{4} \wedge e^{3} \wedge e^{5} \wedge e^{8} \\
& +e^{4} \wedge e^{7} \wedge e^{1} \wedge e^{8}+e^{6} \wedge e^{7} \wedge e^{3} \wedge e^{8}+e^{5} \wedge e^{7} \wedge e^{2} \wedge e^{8}+e^{4} \wedge e^{5} \wedge e^{6} \wedge e^{7} \\
& +e^{2} \wedge e^{3} \wedge e^{7} \wedge e^{4}+e^{1} \wedge e^{3} \wedge e^{5} \wedge e^{7}+e^{1} \wedge e^{3} \wedge e^{5} \wedge e^{7}+e^{1} \wedge e^{2} \wedge e^{7} \wedge e^{6} \\
& +e^{2} \wedge e^{3} \wedge e^{5} \wedge e^{6}+e^{1} \wedge e^{2} \wedge e^{4} \wedge e^{5}+e^{1} \wedge e^{3} \wedge e^{4} \wedge e^{6}
\end{aligned}
$$

where $e^{\alpha}, \alpha=1, \ldots, 8$ is an orthonormal frame (the metric is $\sum_{i} e^{i} \otimes e^{i}$ in this frame), is torsion free, i.e., if it is closed

$$
d \Psi=0 .
$$

In this case, the manifold is Ricci flat. The Cayley form $\Psi$ is self-dual and, in addition, it is invariant under $\operatorname{Spin}(7)$. It is the singlet in the decomposition of the $\mathbf{3 5}_{+}$in the non-standard embedding of $S O(7)$ in $S O(8)$ given in eq.(16) according to which

$$
35_{v}=35, \quad 35_{+}=1+7+27, \quad 35_{-}=35 .
$$

In order to prove now that manifolds which satisfy eq.(18) have $\operatorname{Spin}(7)$ holonomy, let us observe that the Cayley four-form $\Psi$ can actually be written as

$$
\Psi=\frac{1}{4 !} \Psi_{\alpha \beta \gamma \delta} e^{\alpha} \wedge e^{\beta} \wedge e^{\gamma} \wedge e^{\delta}
$$

It is not difficult to verify using eq.(9) and the structure equations

$$
d e^{\alpha}+\omega^{\alpha}{ }_{\beta} e^{\beta}=0, \quad \omega_{\beta}^{\alpha}=\omega_{\beta \mu}^{\alpha} d x^{\mu}
$$

that if the spin connection satisfies eq.(18) the Cayley form $\Psi$ will be closed. Thus, manifolds with connection satisfying the self-duality condition (18) are $\operatorname{Spin}(7)$ Ricci flat manifolds. In addition, such manifolds admit covariantly constant spinors. Indeed, for an $S O(8)$ spinor $\eta$ with

$$
D_{\mu} \eta=\left(\partial_{\mu}-\frac{1}{4} \omega_{\alpha \beta \mu} \Gamma^{\alpha \beta}\right) \eta
$$


where $\alpha, \beta, \ldots=1, \ldots, 8$ are world indices, $\mu, \nu, \ldots=1, \ldots, 8$ are curved space indices and $\omega_{\alpha \beta}$ satisfy eq.(18), one may easily verify that $\eta$ is necessarily right-handed and

$$
D_{\mu} \eta=\partial_{\mu} \eta^{A}-\frac{1}{2} \omega_{\alpha \beta \mu} G^{\alpha \beta} \eta^{A}
$$

where $G^{\alpha \beta}$ has been defined in eq.(14). Thus, $\eta$ is covariantly constant $D_{\mu} \eta=0$ if it is a constant spinor and satisfies eq.(15).

Note also that the integrability condition of eq.(23) is

$$
R_{\alpha \beta \mu \nu} \Gamma^{\alpha \beta} \eta^{A}=0
$$

from which follows, after multiplication with $\Gamma^{\mu}$, that spaces of $\operatorname{Spin}(7)$ holonomy are indeed Ricci flat.

\section{The Octonionic Gravitational Instanton}

We have just seen that a systematic way to construct manifolds of $\operatorname{Spin}(7)$ holonomy is provided by solving eq.(18). Selecting one direction, say the eighth, eq.(18) is written as

$$
\omega_{r}^{8}=-\frac{1}{2} \psi_{r p q} \omega^{p q}
$$

The rest of the equations, namely,

$$
\omega_{q}^{p}=\frac{1}{2} \psi_{q r s}^{p} \omega^{r s}-\psi_{q r}^{p} \omega^{r 8},
$$

are automatically satisfied if eq.(26) holds. The self-duality conditions are then explicitly written as

$$
\begin{array}{ll}
\omega_{81}=-\left(\omega_{23}+\omega_{65}+\omega_{47}\right), & \omega_{82}=-\left(\omega_{31}+\omega_{46}+\omega_{57}\right), \\
\omega_{83}=-\left(\omega_{12}+\omega_{54}+\omega_{67}\right), & \omega_{84}=-\left(\omega_{62}+\omega_{35}+\omega_{71}\right), \\
\omega_{85}=-\left(\omega_{16}+\omega_{43}+\omega_{72}\right), & \omega_{86}=-\left(\omega_{51}+\omega_{24}+\omega_{73}\right), \\
\omega_{87}=-\left(\omega_{14}+\omega_{36}+\omega_{25}\right), &
\end{array}
$$


We will assume that the metric of the $\operatorname{Spin}(7)$-holonomy manifold $X$ is of the form

$$
d s^{2}=f(r)^{2} d r^{2}+g(r)^{2}\left(d \mu^{2}+\frac{1}{4} \sin ^{2} \mu \Sigma_{i}^{2}\right)+h(r)^{2}\left(\sigma_{i}-A_{i}\right)^{2}
$$

where $\Sigma_{i}$ and $\sigma_{i}, i=1,2,3$ are $S U(2)$ left-invariant one-forms satisfying

$$
d \Sigma_{i}=-\frac{1}{2} \epsilon_{i j k} \Sigma_{j} \wedge \Sigma_{k}, \quad d \sigma_{i}=-\frac{1}{2} \epsilon_{i j k} \sigma_{j} \wedge \sigma_{k}
$$

and

$$
A_{i}=\cos ^{2} \frac{\mu}{2} \Sigma_{i}
$$

In terms of the angular coordinates $\alpha, \beta, \gamma, \theta, \phi, \psi$ with $0<\alpha, \theta \leq \pi, \quad 0<\beta, \phi \leq 2 \pi$, $0<\gamma, \psi \leq 4 \pi$, the $S U(2)$ left-invariant one-forms $\Sigma_{i}, \sigma_{i}$ are explicitly given by

$$
\begin{aligned}
& \Sigma_{1}=\cos \gamma d \alpha+\sin \gamma \sin \alpha d \beta, \quad \Sigma_{2}=-\sin \gamma d \alpha+\cos \gamma \sin \alpha d \beta, \quad \Sigma_{3}=d \gamma+\cos \alpha d \beta, \\
& \sigma_{1}=\cos \psi d \theta+\sin \psi \sin \theta d \phi, \quad \sigma_{2}=-\sin \psi d \theta+\cos \psi \sin \theta d \phi, \quad \sigma_{3}=d \psi+\cos \theta d \phi,
\end{aligned}
$$

The surfaces $r=$ const. have the topology of $S U(2)$-bundle over $S^{4}$ with $A_{i}$ the $k=1$ $S U(2)$ instanton on $S^{4}$. The isometry of $X$ for generic $g(r), h(r)$ are $S O(5) \times S U(2)$. In particular, if $g(r)=h(r)$ the isometry group is enlarged to $S O(8)$ since in this case the surfaces $r=$ const. are round seven-spheres $S^{7}$ written in their Hopf-fibered form. It should be noted that the ansantz for the metric (29) is similar to the one for the EguchiHanson $4 \mathrm{~d}$ gravitational instanton where the Hopf-fibration of the $S^{7}$ is replaced with the corresponding fibration of $S^{3}$.

Employing the orthonormal base

$$
\begin{aligned}
e^{i}=\frac{1}{2} g(r) \sin \mu \Sigma_{i}, & e^{\hat{i}}=h(r)\left(\sigma_{i}-\cos ^{2} \frac{\mu}{2} \Sigma_{i}\right), \\
e^{7}=f(r) d r, \quad e^{8} & =g(r) d \mu,
\end{aligned}
$$

where $i=1,2,3$ and $\hat{i}=4,5,6=\hat{1}, \hat{2}, \hat{3}$, we find that the spin connection is

$$
\omega_{i j}=-\left(\frac{1}{g(r) \sin \mu} e^{k}+\frac{h(r)}{2 g(r)^{2}} e^{\hat{k}}\right) \epsilon_{i j k}, \quad \omega_{\hat{i} \hat{j}}=-\left(\frac{1}{2 h(r)} e^{\hat{k}}+\frac{2 \cos ^{2} \mu / 2}{g(r) \sin \mu} e^{k}\right) \epsilon_{i j k}
$$




$$
\begin{array}{rlrl}
\omega_{i \hat{j}}=\frac{h(r)}{2 g(r)^{2}} \epsilon_{i j k} e^{k}+\frac{h(r)}{2 g(r)^{2}} \delta_{i j} e^{8}, & \omega_{8 i} & =-\frac{1}{g(r)} \cot \mu e^{i}-\frac{h(r)}{2 g(r)^{2}} e^{\hat{i}}, \\
\omega_{8 \hat{i}} & =-\frac{h(r)}{2 g(r)^{2}} e^{i}, & \omega_{87} & =\frac{g(r)^{\prime}}{g(r) f(r)} e^{8}, \\
\omega_{i 7} & =\frac{g(r)^{\prime}}{g(r) f(r)} e^{i}, & \omega_{\hat{i} 7} & =\frac{h(r)^{\prime}}{h(r) f(r)} e^{\hat{i}},
\end{array}
$$

with $^{\prime}=d / d r$. Then, using the components of the connection, we find that eq.(28) is satisfied if $h(r), g(r)$ obey the differential equations

$$
\frac{h(r)^{\prime}}{h(r) f(r)}+\frac{1}{2 h(r)}-\frac{h(r)}{g(r)^{2}}=0, \quad \frac{g(r)^{\prime}}{g(r) f(r)}+\frac{3 h(r)}{2 g(r)^{2}}=0 .
$$

We may use the reparametrization invariance of the metric under $r \rightarrow r^{\prime}=r^{\prime}(r)$ to fix one of the three functions $h(r), g(r)$ and $f(r)$. In particular, choosing

$$
g(r)^{2}=\frac{9}{20} r^{2}
$$

we find after solving eq.(34) that

$$
h(r)^{2}=\frac{1}{5} g(r)^{2}\left(1-\left(\frac{m}{r}\right)^{10 / 3}\right), \quad f(r)^{2}=\frac{1}{1-\left(\frac{m}{r}\right)^{10 / 3}}
$$

where $m$ is an integration constant taken as the moduli. Thus, the full metric (29) turns out to be

$$
d s^{2}=\frac{d r^{2}}{1-\left(\frac{m}{r}\right)^{10 / 3}}+\frac{9}{20} r^{2}\left(d \mu^{2}+\frac{1}{4} \sin ^{2} \mu \Sigma_{i}\right)+\frac{9}{100} r^{2}\left(1-\left(\frac{m}{r}\right)^{10 / 3}\right)\left(\sigma_{i}-\cos ^{2} \frac{\mu}{2} \Sigma_{i}\right)^{2}
$$

It resembles the Eguchi-Hanson metric with moduli $m$, but in the present case we have a radial fall off rate $r^{-10 / 3}$, instead of $r^{-4}$ in Eguchi-Hanson, because of the eight-dimensional nature of our solution.

One may easily verify that the apparent singularity at $r=m$ is a removable bolt singularity. Indeed, near $r=m$, the metric (37) at $\mu, \alpha, \beta, \gamma=$ const., takes the form

$$
d s^{2} \sim \frac{9}{25}\left(d \rho^{2}+\frac{1}{4} \rho^{2} \sigma_{i}^{2}\right)
$$


with $\rho^{2}=r^{2}\left(1-(m / r)^{10 / 3}\right)$ which is the metric on $\mathbf{R}^{4}$ in polar coordinates. Thus, the topology of $X$ is locally of the form $\mathbf{R}^{4} \times S^{4}$ and the singularity at $r=m$ is just a coordinate singularity. Asymptotically the metric (37) takes the form

$$
d s^{2}=d r^{2}+\frac{9}{20} r^{2}\left(d \mu^{2}+\frac{1}{4} \sin ^{2} \mu \Sigma_{i}+\frac{1}{5}\left(\sigma_{i}-\cos ^{2} \frac{\mu}{2} \Sigma_{i}\right)^{2}\right),
$$

and thus the boundary at infinity is the squashed $S^{7}$ [14].

Finally we note for completeness that the bolt structure and the asymptotic behaviour of the metric are insensitive to the value of the free parameter $m$. This should be compared to the similar behaviour of the Eguchi-Hanson metric in four dimensions, which for $m=0$ reduces to the flat space metric in polar coordinates modulo global issues that arise from identifications in the allowed range of the Euler angles resulting in $S^{3} / Z_{2}$ at infinity.

\section{Brane Solutions}

The solution (37) we found above can be lifted to appropriate brane solutions in tenand eleven-dimensional supergravity. In particular, the octonionic gravitational instanton may be taken as the transverse space of a fundamental string in string theory or of a fundamental membrane in M-theory. It can also be considered as a domain-wall solution (eight-brane) in massive IIA supergravity. In all cases, these brane solutions preserve the minimum amount of supersymmetry since there exists only one covariant constant spinor on the octonionic gravitational instanton. Thus, the brane solutions we will describe in the following are 1/16 BPS states.

\section{- Type II Octonionic Fundamental String}

We consider ten-dimensional backgrounds of the form $M^{2} \times N^{8}$ with metric

$$
d s^{2}=e^{2 A}\left(-d t^{2}+d x^{2}\right)+e^{2 B} g_{m n} d y^{m} d y^{n}
$$


where $y^{m}, m=1, \ldots, 8$ are coordinates on the transverse space $N^{8}, A=A(y), B=B(y)$ and $g_{m n}$ is the metric on $N^{8}$. Similarly, we assume that the dilaton $\phi=\phi(y)$ and the only non-vanishing component of the antisymmetric NS-NS two-form $B_{M N}$ is $B_{t x}=-e^{C(y)}$. Then, we find that the fermionic shifts equal to

$$
\begin{aligned}
\delta \psi_{M} & =D_{M} \epsilon+\frac{1}{96} e^{-\phi / 2}\left(\Gamma_{M}^{N P Q}-9 \delta_{M}^{N} \Gamma^{P Q}\right) H_{N P Q} \epsilon \\
\delta \lambda & =-\frac{1}{2 \sqrt{2}} \Gamma^{m} \partial_{m} \phi \epsilon+\frac{1}{24 \sqrt{2}} e^{-\phi / 2} \Gamma^{M N P} H_{M N P} \epsilon
\end{aligned}
$$

vanish if

$$
A=\frac{3 \phi}{4}, \quad B=-\frac{\phi}{4}, \quad C=2 \phi
$$

and the transverse space $N^{8}$ admits a covariant constant spinor. Finally, from the field equations, we obtain that $e^{-2 \phi}$ is harmonic in $N^{8}$, i.e.,

$$
\nabla^{2} e^{-2 \phi}=0
$$

Recall at this point that our octonionic gravitational instanton admits a covariant constant spinor and so it can be taken as a solution for the transverse space $N^{8}$. In this case eq. (43) reduces to

$$
\frac{1}{r^{7}} \partial_{r}\left(r^{7}\left[1-\left(\frac{m}{r}\right)^{10 / 3}\right] \partial_{r} e^{-2 \phi}\right)=0 .
$$

The solution is described in terms of the Gauss hypergeometric function ${ }_{2} F_{1}(a, b ; c, z)$ as

$$
e^{-2 \phi(r)}=e^{-2 \phi_{\infty}}-\frac{\Lambda_{2}}{r^{6}}{ }_{2} F_{1}\left(1, \frac{9}{5} ; \frac{14}{5},(m / r)^{10 / 3}\right)
$$

where $\Lambda_{2}$ is a constant proportional to the string tension $T_{2}$, using the integral representation of ${ }_{2} F_{1}(a, b ; c, z)$

$$
{ }_{2} F_{1}(a, b ; c, z)=\frac{\Gamma(c)}{\Gamma(b) \Gamma(c-b)} \int_{0}^{1} t^{b-1}(1-t)^{c-b-1}(1-z t)^{-a} d t, \quad \text { Re } c>\operatorname{Re} b>0 .
$$

To compare it with other results in the literature, we note that a heterotic octonionic string was constructed in [15] with $(0,1)$ supersymmetry on its world-volume. In that case, 
however, the Bianchi identity for $H_{M N P}$ was solved by introducing appropriate self-dual eight-dimensional gauge fields; for the relevant technical details we refer the reader to the literature [16]. Hence, the solution we describe in this paper is different.

\section{- Octonionic Fundamental Membrane}

We may also lift the octonionic gravitational instanton in the eleven-dimensional supergravity. Here, the bosonic fields in the graviton multiplet is the graviton and the antisymmetric three-form $A_{M N P}$. We will thus consider an eleven-dimensional background of the form $M^{3} \times N^{8}$ with metric

$$
d s^{2}=e^{2 A}\left(-d t^{2}+d x_{1}^{2}+d x_{2}^{2}\right)+e^{2 B} g_{m n} d y^{m} d y^{n},
$$

and non-vanishing components for $A_{M N P}$, namely $A_{t x_{1} x_{2}}=e^{-C}$, where $A=A(y), B=$ $B(y)$ and $C=C(y)$ as before. In this case, the gravitino shift

$$
\delta \psi_{M}=D_{M} \epsilon+\frac{1}{288}\left(\Gamma_{M}^{N P Q R}-8 \delta_{M}^{N} \Gamma^{P Q R}\right) F_{N P Q R} \epsilon
$$

vanish if there exists a covariant constant spinor on $N^{8}$ and

$$
A=\frac{2}{3} C, \quad B=-\frac{1}{3} C .
$$

Thus, we may choose for $N^{8}$ the octonionic gravitational instanton as before. In addition, the field equations are satisfied if $e^{-C}$ is a harmonic function on $N^{8}$. In this case we find

$$
e^{-C(r)}=e^{-C_{\infty}}-\frac{\Lambda_{3}}{r^{6}}{ }_{2} F_{1}\left(1, \frac{9}{5} ; \frac{14}{5},(m / r)^{10 / 3}\right)
$$

where now $\Lambda_{3}$ is proportional to the membrane tension $T_{3}$.

The fundamental octonionic membrane we have just constructed is completely different from the octonionic membrane constructed in [17]. We only note that the latter is not supersymmetric and furthermore it has a non-trivial $F_{m n p q}$ proportional to the self-dual tensor in eq.(7). Hence our result yields a new configuration in eleven-dimensional supergravity. 


\section{Discussion}

Summarizing, we have shown that eight-dimensional gravitational instantons can be constructed as solutions of self-duality equations for the spin connection. The octonions, which are integral part of our method, yield in this fashion Ricci flat manifolds with holonomy in $\operatorname{Spin}(7)$. We have also presented an explicit solution with isometry group $S O(5) \times S U(2)$ having a bolt singularity and the asymptotic structure of the squashed seven-sphere; it can be regarded as a higher dimensional analogue of the Eguchi-Hanson space. Also, because of supersymmetry, we can elevate our solution to ten and eleven dimensions in order to obtain octonionic fundamental string and membrane configurations respectively which are 1/16 BPS states.

The present framework can be developed further by considering eight-dimensional solutions with $S U(2)$ isometry and attempt a classification of the corresponding gravitational instanton symmetries in analogy with four dimensions [2]. In the four dimensional case one considers the action of an one-parameter isometry group $U(1)$ and classifies its fixed points into two different types: isolated points called nuts and 2-surfaces (spheres) called bolts. Then, performing a dimensional reduction of Einstein's equations with respect to the orbits of the $U(1)$ isometry group to three dimensions, one exhibits a duality between the electric and the magnetic aspects of gravity (corresponding to bolts and nuts respectively) via the Ehlers transform. Of course, certain solutions might exhibit more isometries which are not of general value; for instance the Eguchi-Hanson space has an additional $S O(3)$ isometry apart from the $U(1)$. In eight dimensions, although the particular solution we found has an additional $S O(5)$ isometry apart from $S U(2)$, we think that focusing on more general classes of solutions with $S U(2)$ isometry are worth studying further. Selecting gravitational instantons with $S U(2)$ isometry in eight dimensions is also quite natural from the point of view of Hopf fibrations, as is the selection of gravitational instantons with $U(1)$ isometry in four dimensions. 
In the reduction of an eight dimensional theory to five dimensions using the orbits of the corresponding isometry group, $S^{3}$, we are naturally led to the notion of "nut potential" as an antisymmetric 2-form field that dualizes the $S U(2)$ gauge field connection of the metric. The reasoning is the same one that yields an axion-like scalar function as nut potential in four-dimensional spaces with $U(1)$ isometry. It will be interesting to carry out this line of investigation in the present case and see whether $M 2$ and $M 5$ branes, when appropriately taken in eleven-dimensional supergravity, provide the analogue of bolts and nuts in establishing various electric and magnetic aspects of eight dimensional gravitational instantons. We hope that in this way, apart for improving our conceptual understanding of the general problem, we will also be able to find more explicit instanton solutions as in four dimensions. We plan to report on these issues elsewhere.

Note Added: We have been informed by B. Acharya (private communication) that he has also employed the squashed $S^{7}$ in searching for $\operatorname{Spin}(7)$-holonomy manifolds (unpublished work). We also became aware after circulating the first draft of our paper that the particular metric (37) was constructed some time ago by solving directly Einstein's equations in eight dimensions [18]; we thank G. Gibbons and C. Pope for bringing their paper to our attention. Their study was based on second order differential equations rather than the first order self-duality equations (18), and (in a sense) made explicit earlier mathematical results on the construction of complete metrics with $\operatorname{Spin}(7)$ holonomy [19]. We thank D. Morrison for also pointing out the relevance of ref. 19 to the general framework we have adopted here. 


\section{References}

[1] S.W. Hawking, Phys. Lett. A 60 (1977) 81.

[2] G.W. Gibbons and S.W. Hawking, Comm. Math. Phys. 66 (1979) 291.

[3] T. Eguchi, P.B. Gilkey and A.J. Hanson, Rhys. Rep. 66 (1980) 213.

[4] T. Eguchi and A.J. Hanson, Phys. Lett. B 74 (1978) 249;

G.W. Gibbons and S.W. Hawking, Phys. Lett. B 78 (1978) 430.

[5] A. Trautman, Int. J. Phys. 16 (1977) 561.

[6] E. Floratos and A. Kehagias, Phys. Lett. B 427 (1998) 283, hep-th/9802107.

[7] R. Bryant, Ann. Math. 126 (1987) 525.

[8] B.S. Acharya and M. O'Loughlin, Phys. Rev. D 55 (1997) 4521, hep-th/9612182.

[9] D. Joyce, Invent. Math. 123 (1996) 507.

[10] M. Günaydin and F. Gürsey, J. Math. Phys. 14 (1973) 1651;

R. Dündarer, F. Gürsey and C.H. Tze, J. Math. Phys. 25 (1984) 1496.

[11] B. de Wit and H. Nicolai, Nucl. Phys. B 231 (1984) 506

[12] A. Chakrabarti, Phys. Lett. B 172 (1986)175.

[13] S.M. Salamon, "Riemannian geometry and holonomy groups", Pitman Res. Notes in Math. 201, Longman, 1989.

[14] M.A. Awada, M.J. Duff and C.N. Pope, Phys. Rev. Lett. 50 (1983) 294; see also, M.J. Duff, B.E.W. Nillson and C.N. Pope, Phys. Rep. 130 (1986) 1.

[15] J.A. Harvey and A. Strominger, Phys. Rev. Lett. 66 (1991) 549. 
[16] D.B. Fairlie and J. Nuyts, J. Phys. A 17 (1984) 2867;

S. Fubini and H. Nicolai, Phys. Lett. B 155 (1985) 369;

B. Grossman, T. Kephart and J.D. Stasheff, Comm. Math. Phys. 96 (1984) 431;

M. Gunaydin and H. Nicolai, Phys. Lett. B 351 (1995) 169; ibid B 376 (1996) 329.

[17] M.J. Duff, J.M. Evans, R.R. Khuri, J.X. Lu and R. Minasian, Phys. Lett. B 412 (1997) 281, hep-th/9706124.

[18] G.W. Gibbons, D.N. Page and C.N. Pope, Commun. Math. Phys 127 (1990)529.

[19] R.B. Bryant and S.M. Salamon, Duke Math. J. 58 (1989)829. 